\title{
Screening and Breeding of Kluyveromyces Strains for Production Pectinolytic Activity
}

\author{
Luciani Tatsch Piemolini-Barreto ${ }^{1,2 *}$, Regina Vasconcellos Antônio ${ }^{2}$ and Sergio Echeverrigaray ${ }^{3}$ \\ ${ }^{1}$ University of Caxias do Sul, Brazil \\ ${ }^{2}$ Department of Chemical and Food Engineering, Federal University of Santa Catarina, Brazil \\ ${ }^{3}$ Institute of Biotechnology, University of Caxias do Sul, 1352, Brazil
}

Submission: March 15, 2017 ;Published: April 24, 2017

*Corresponding author: Luciani Tatsch Piemolini-Barreto, Center of Exact Sciences and Technologies, University of Caxias do Sul, 1352, Caxias do Sul, RS 95001-970, Department of Chemical and Food Engineering, Federal University of Santa Catarina, 16 Florianópolis, SC 88040-900, Brazil, Fax: +55-54-32182149; Email: ltpbarre@ucs.br

\begin{abstract}
Polygalacturonases are pectinolytic enzymes that have important industrial application inextraction and clarification of fruit juices and in process winemaking. Kluyveromyces strains were screened for pectinase production. The production of pectinase by Kluyveromyces marxianus was evaluated at different concentrations of pectin and glucose. All the strains grew on citric pectin as sole carbon source. Breeding and selection of pectinolytic segregants resulted in important increase on extracellular total pectinolytic activity. The evaluation of the best strain (NRRL-Y-7571) under different condition showed that oxygen depletes polygalacturonases activity, pectin acts as an inducer, and glucose stimulates grown but did not affect enzyme production. The kinetic study showed that the highest enzyme activity was achieved at $48 \mathrm{~h}$ of fermentation. The cultivation of K. marxianus NRRL-Y-7571 in 2\% pectin medium under a restricted oxygen condition resulted in the highest extracellular total pectinolytic activity (118.5 U.mL-1). Such studies are very scarce in the literature and should be helpful to understand the true potentialities of pectinases obtained by yeast in industrial applications.
\end{abstract}

Keywords: Kluyveromyces marxianus; Yeast breeding; Enzymes; Pectinolytic activity

\section{Introduction}

Enzymes that act on pectic substances by hydrolysis, trans elimination and de-esterification reactions, which contribute to the firmness and structure of plant cells, are known as pectinolytic enzymes or pectinases [1]. Based on the pectin degradation mechanism they are classified mainly into pectinesterase (PME), pectinlyase (PL) and polygalacturonase (PG). Among them, polygalacturonase hydrolyzes -1, 4 glycosidic linkages of polygalacturonic acid chain and releases galacturonic acid residues [2]. Pectinolytic enzymes are particularly important for the food industry, mainly in the clarification of fruit juices [3] and wine industry [4]. Pectinases are of great importance for clarification and viscosity reduction in fruit juices. Clarification is achieved by pectin hydrolysis, leading to decreased viscosity and agglomeration of particulate matter that may be removed or sufficiently reduced by filtration or centrifugation [5].

The production of pectinases usually relies on different species of filamentous fungi, bacteria and yeasts [6]. Filamentous fungi are the most frequently used microorganisms in the enzyme industry because at least $90 \%$ are produced extracellularly
[7]. Pectinases can be produced at different concentrations by strains of filamentous fungi belonging to Aspergillus genus and certain Aspergillus species have been characterized by the types of pectinolytic enzymes they produce [8]. However, yeasts represent another alternative source of this enzyme, and most of them are able to provide high yields of pure enzymes [9].

Yeasts present advantages over filamentous fungi, mainly because yeasts are unicellular organisms, the growth medium does not require the addition of inducers [6], and usually yeasts do no secret pectin esterase [10], consequently their pectinases can be used to process fruit juice and wine without releasing methanol [11,12]. In addition, cloning andgene manipulation may improve enzyme production, which could foster its industrial application on a large scale [13]. The production of pectinolytic enzymes depends on microbial strains and production conditions. The production of pectinases by fungi and yeasts is affected by several physical parameters (e.g. temperature,pH, oxygen supply) and chemical (culture medium composition) [14]. 
In general, the fermentative production of these enzymes requires special attention to the composition of the culture medium, since it can contain components involved in the induction, repression or inhibition of enzyme formation and secretion of every strain of interest $[7,15]$. According to Kouhoundé et al. [16], the production of PG by microorganisms is especially dependent on the composition of the cultivation media (glucose and pectin) and the variation of $\mathrm{pH}$ during the process. Polygalacturonase induction by pectin was observed in different yeast species, including Saccharomyces cerevisiae $[17,18]$ and Kluyveromyces marxianus [19]. Nakagawa et al. [20] described the yeast Candida boidinii S2 was found to be able to grow on pectin or polygalacturonate as a carbon source. Conversely, Schwan \& Rose [21] did not evidence polygalacturonase induction by pectin in the Kluyveromyces marxianus CCT 3172 isolate, indicating that pectin induction may be strain specific. Considering the promising opportunities that Kluyveromyces species might offer for the food industry, the present study was to select highly pectinolytic strains of Kluyveromyces sp., and to evaluate the effect of pectin and glucose concentrations on the production total pectinolytic by the selected strain.

\section{Materials and Methods}

\section{Yeast strains and breeding}

In this study, 19 Kluyveromyces strains were evaluated. These strains include 11 isolates obtained from the National Center for Agricultural Utilization Research (NRRL), the American Type Culture Collection (ATCC), the Instituto Zimotécnico (ESALQ, USP, Brazil), and the Industrial Technology Foundation (FTI, SP, Brazil), four auxotrophic mutants, a hybrid, and three meiotic segregants selected based on their growth onminimal medium with pectin as sole carbon source. $K$. marxianus hybrid was obtained by mass mating and selection by auxotrophic marks complementation onminimal medium (2\% glucose, $0.67 \%$ YNB without aminoacids, $2 \%$ agar). Sporulation was induced on raffinose-acetate medium, vegetative cells eliminated by diethyl ether treatment [22], and spores plated on pectin-YP medium (1 $\%$ yeast extract, $2 \%$ peptone, $1 \%$ pectin, $2 \%$ agar).

Media and Experimental conditions for the selection and production of enzymes Pectinolytic strains were selected by the method proposed by McKay [23]. Briefly, the microorganisms were inoculated in YNB-pectin (1\% citric pectin (ESKISA S.A., Brazil), $0.5 \%$ yeast extract, $0.67 \%$ yeast nitrogen base (YNB), and $2 \%$ agar, pH 5.0), and grown at $30{ }^{\circ} \mathrm{C}$ for $96 \mathrm{~h}$. The plates were overlaid with an aqueous solution of ruthenium-red $(0.05$ $\%)$, incubated for $5 \mathrm{~min}$, and then washed with distilled water. The clear halos of pectin degradation were measured, and the relative pectinolytic activity (RPA) was calculated as a relation between the diameter of the degradation halo and the colony diameter.

To evaluate the extracellular pectinolytic activity, yeast strains were grown for $48 \mathrm{~h}$ at $28{ }^{\circ} \mathrm{C}$ and $150 \mathrm{rpm}$ in YEPD (2
$\%$ glucose, $2 \%$ peptone, $1 \%$ yeast extract, $\mathrm{pH} 6.8$ ), and used to inoculate $\left(2 \times 107\right.$ cells. $\left.\mathrm{mL}^{-1}\right) 50 \mathrm{ml}$ of citric-pectin broth $(0.5$ $\%$ yeast extract, $1 \%$ citric pectin (ERKISA S.A., Brazil), $0.67 \%$ yeast nitrogen base, $\mathrm{pH}$ 5.0). Cultures were maintained at 28 ${ }^{\circ} \mathrm{C}$ for $48 \mathrm{~h}$, under static conditions. The solution thus obtained was then centrifuged at $5000 \mathrm{~g}$ for $10 \mathrm{~min}$. The supernatant (enzymatic extract) was kept at $4{ }^{\circ} \mathrm{C}$. To evaluate the influence of culture medium composition on yeast growth and total pectinolytic activity production, four media with different concentrations of glucose and citric pectin (ERKISA S.A., Brazil) were formulated: (1) $1 \%$ pectin, (2) $2 \%$ pectin; (3) $1 \%$ pectin and $1 \%$ glucose, and (4) $1 \%$ glucose. From the previous studies [1], these carbon sources were incorporated on the basal medium $(0.67$ \% Yeast Nitrogen Base, $0.5 \%$ Yeast Extract, pH 5.0). The culture media (50mL) were inoculated ( $2 \times 107$ cells. $\mathrm{mL}^{-1}$ final concentration) from a two day old culture in YEPD at $28^{\circ} \mathrm{C}$ and $150 \mathrm{rpm}$. The experiments were conducted at $28^{\circ} \mathrm{C}$ for $96 \mathrm{~h}$, under static conditions. Samples were collected every $8 \mathrm{~h}$, centrifuged (10min, $10000 \mathrm{rpm}$ ), and the supernatant (crude extracts) stored at $4{ }^{\circ} \mathrm{C}$. The experiments were conducted twice with three replications of each treatment [24].

\section{Analytical methods}

The total pectinolytic activity was estimated by measuring the release of reducing groups by using the dinitrosalicilic (DNS) method [24] and adapted to small volumes. Briefly, the crude extract $(50 \mu \mathrm{L})$ was mixed with $50 \mu \mathrm{L}$ of polygalacturonic acid solution ( $0.1 \%$ in $25 \mathrm{mM}$ acetate buffer pH 5.0) in PCR plates with 96 holes $(200 \mu \mathrm{L})$. The plates were incubated at $40{ }^{\circ} \mathrm{C}$ for $1 \mathrm{~h}$ in a Research Thermal Cycler model PTC 100, and the reaction was stopped by adding $60 \mu \mathrm{L}$ of DNS. The plates were again placed in the thermal cycles and kept at $95{ }^{\circ} \mathrm{C}$ for $15 \mathrm{~min}$. The volume was then completed to $200 \mu \mathrm{L}$ with distilled water. Samples $(150 \mu \mathrm{L})$ were transferred to microplates and the absorbance (595nm) was evaluated in a Biochrom Libra S12 microplate reader. One unit of activity was defined as the amount of enzyme required to release $1 \mu \mathrm{mol}$ (equivalent of galacturonic acid) of reducing groups per minute. Quantification of the reducing groups was performed by comparison with a standard curve of galacturonic acid $(0-80 \mu \mathrm{g})$ under the same conditions. All the tests were made in triplicate. The concentration of reducing groups in the culture supernatant was determined by the DNS method described above, but using water instead of polygalacturonic acid solution, and incubating the samples directly at $95{ }^{\circ} \mathrm{C}$ for $15 \mathrm{~min}$. A standard curve of glucose ( 0 to $\left.10 \mathrm{~g} . \mathrm{L}^{-1}\right)$ was used. Yeast growth was estimated by optical density at a wavelength of $600 \mathrm{~nm}$.

\section{Statistical analysis}

Data were submitted to one-way analysis of variance (ANOVA), and means comparison by the Tuckey's test with a probability level below $5 \%(\mathrm{p}<0.05)$. The analyses were conducted on the PASW 18.0.0 statistics. 


\section{Results and Discussion}

\section{Selection of pectinolytic strains}

Initially, pectinolytic strains were selected by the method of pectin degradation calculated as a relation between the diameter of the degradation halo and the colony diameter. Among the 19 Kluyveromyces strains evaluated, 16 (84\%) showed pectinolytic activity on agar plates. The three strains (NRRL-Y-8278, NRRL-Y-1195, NRRL-Y-1207) that did not exhibited halo, grew on YNB-pectin medium, indicating that there are able to use pectin as sole carbon source. These strains showed just a basal extracellular total pectinolytic activity (1.07 to 1.69 U.mL $\mathrm{m}^{-1}$ ) when compared with the other strains. A large and significant variation was observed on the total pectinolytic activity among the Kluyveromyces strains evaluated, and a significant correlation was detected between relative activity (halo) and PG activity $(R=0.951)$, indicating that relative activity can be used for strains selection. The highest extracellular pectinolytic activity was detected on NRRL-Y-7571 (62.79 U.mL-1), followed by NRRL-Y-6373 (30.88 U.mL-1). The pectinolytic activity detected in these strains were higher than those previously reported in different wild yeast species, including Kluyveromyces [25,26], and a recombinant $S$. cerevisiae strain [13].

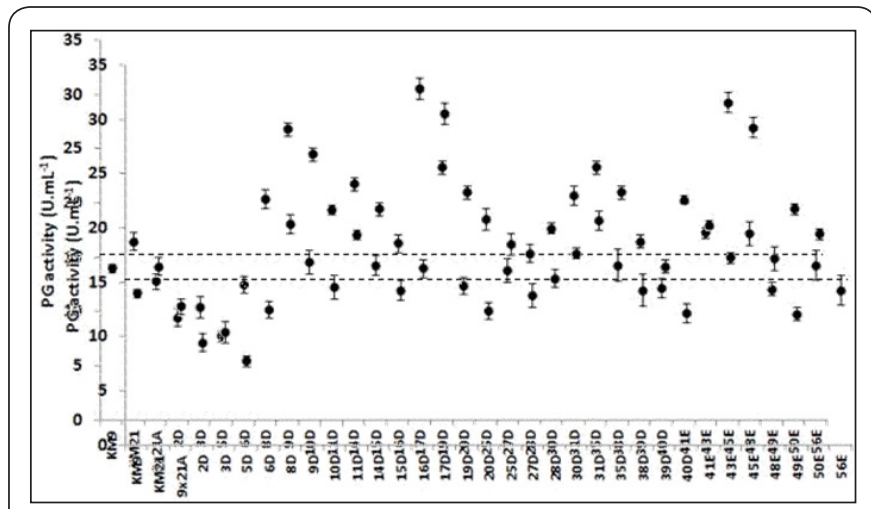

Figure 1: Extracellular total pectinolytic activity of parental strains, hybrid and selected segregants. Dotted line indicates the mean pectinolytic activity ( $P G$ activity) of the parental strains $\mathrm{KM} 9$ and KM21.

The auxotrophic mutants KM9 (met-) and KM21 (arg-) were crossed producing the $9 \times 21 \mathrm{~A}$ hybrid. This hybrid was sporulated and 30 segregants were initially selected by their pectinolytic halos on YNB-pectin medium. The segregants exhibited a large variation on extracellular pectinolytic activity and $40 \%$ were significantly higher than the parental strains (Figure 1). The pectinolytic activity of the selected segregants 9D, 17D and 45E was $80.5,94.4$ and $99.4 \%$ superior than the average of KM9 and KM21, indicating that conventional breeding methods can be efficiently used to increase extracellular total pectinolytic activity in Kluyveromyces. Effect of medium composition and agitation on the total pectinolytic activity production by Kluyveromyces marxianus NRRL-Y-7571 in the sequence, submerged fermentation runs with media containing 0 and $1 \%(\mathrm{w} / \mathrm{w})$ glucose and citric pectin concentrations of 0,1 , and $2 \%(w / w)$. The aim of these experiments was to test the replacement of a part of the pectin by a simpler and cheaper carbon source for the growth of $K$. marxianus as well as to obtain more information on the induction/repression process of polygalacturonase production by this yeast. Based on the above results, NRRL-Y-7571 was selected for further analysis.

A first experiment in pectin $1 \%$ YNB medium, conducted under constant shaking (150 rpm) and static condition, confirmed the negative effect of dissolved oxygen on total pectinolytic activity production by Kluyveromyces marxianus, with maximum activity of $10.9 \mathrm{U}_{\mathrm{mL}} \mathrm{mL}^{-1}$ in agitated, and 60.8 U.mL $L^{-1}$ in non-agitated cultures, respectively (Figure 2). Our results agree with those reported by Cruz- Guerrero et al. [27], who analyzed dissolved oxygen in the repression of endopolygalacturonase production by Kluyveromyces marxianus. Yeast growth, reduction products variation, and total pectinolytic activity during static cultures of $K$. marxianus NRRL-Y-7571 on four media with different concentrations of glucose and pectin are shown in Figure 3. As can be observed, independent of the culture media, NRRL-Y-7571 showed an initial lag phase of $8 \mathrm{~h}$ followed by a logarithmic growth phase of $8 \mathrm{~h}$ in $1 \%$ pectin to $16 \mathrm{~h}$ on the other media (Figure 3A). Moreover, no differences were observed on yeast growth on the pectin, glucose or pectin/glucose media, indicating that poly galacturonic acid is efficiently metabolized by NRRL-Y-7571. Kluyveromyces growth was accompanied by a decrease on reducing groups in culture media (Figure 3B).

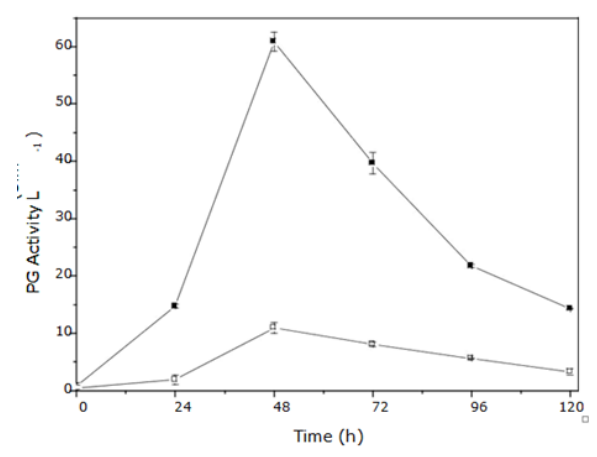

Figure 2: Figure 2: Effect of aeration on the extracellular total pectinolytic activity (PG activity) of K. marxianus NRRL-Y-7571. Static (solid square) and shacked (open square) culture.

The absence of reducing products accumulation in pectin and pectin/glucose media indicates that polygalacturonic acid released by total pectinolytic activity is rapidly incorporated and used by yeast cells. Filamentous fungi D-galacturonic acid metabolism operates by a specific pathway that involves a sequential conversion of D-galacturonic acid to L-galactonate, 2-keto-3-deoxy-L-galactonate, and glycerol and pyruvate [28]. Although conserved in fungi, we were not able to find homologous to fungal D-galacturonic acid reductase and L-galacturonate dehydrogenase, the two initial enzymes in D-galacturonic acid pathway, in Saccharomyces cerevisiae and Kluyveromyces lactis 
genomes, posing an interesting question on the metabolism of D-galacturonic acid residues by pectinolytic yeasts. Besides the carbon source, other medium components must be carefully chosen to achieve good results.

The culture media with an adequate balance of simple carbohydrates and pectin lead to the best production of pectinases in a submerged process. The process was evaluated in medium containing different concentrations of pectin with glucose addition. In the medium without pectin, production of the PG was lower. When using pectin, higher activities of PG were obtained, indicating that the enzyme production by $\mathrm{K}$. marxianus is strongly affected by the presence of this inducer. Higher PG activities were found in media with increased pectin content. The highest total pectinolytic activity of $K$. marxianus NRRL- Y-7571 was obtained in $2 \%$ pectin medium (118.5 U. $\mathrm{mL}-1$ ) (Figure 3C). Independent of the culture media, total pectinolytic activity reached its maximum after $48 \mathrm{~h}$ of culture, when the yeast attained the stationary phase, followed by a low but significant decay (Figure 3C). The association between $K$. marxianus growth and total pectinolytic activity was previously evidenced [25]. The total pectinolytic activity in $1 \%$ glucose medium (36 U.mL ${ }^{-1}$ ) indicates the constitutive production of this enzyme by Kluyveromyces. However, in $1 \%$ glucose plus $1 \%$ pectin medium, the activity was significantly higher $\left(50 \mathrm{U} \cdot \mathrm{mL}^{-1}\right)$, indicating that although constitutive this enzyme is induced by pectin or their products.
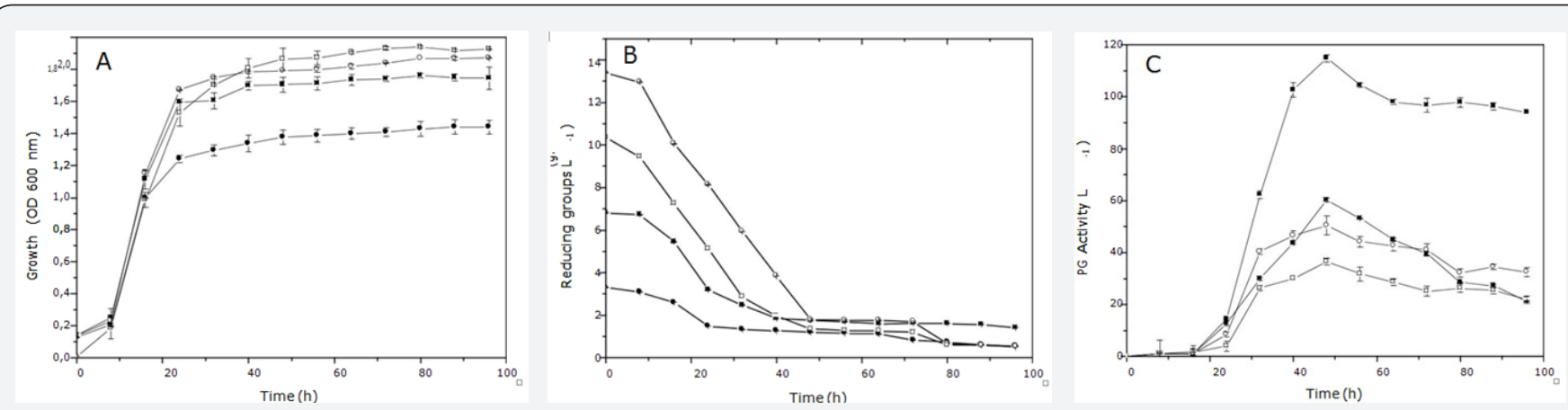

Figure 3: Effect of glucose and pectin on yeast growth and extracellular total pectinolytic activity (PG activity). Glucose $1 \%$ (open square), glucose $1 \%$ with pectin $1 \%$ (open circle), pectin $1 \%$ (solid circle), and pectin $2 \%$ (solid square).

\section{Conclusion}

In summary, the present results showed that the selection and breeding of $K$. marxianus can significantly increase the total pectinolytic activity production, and confirmed that the establishment of adequate culture conditions for specific strains is essential to obtain high pectinolytic extract for industrial purposes.

\section{Acknowledgement}

The authors are grateful to CNPq, CAPES, FAPERGS and the University of Caxias do Sul for providing scholarships and grant that directly or indirectly allowed the development of this work.

\section{References}

1. Kashyap DR, Vohra PK, Chopra S, Tewari R (2001) Applications of pectinases in the commercial sector: a review. Bioresource Technol 77: 215-227.

2. Kant S, Vohra A, Gupta R (2013) Purification and physicochemical properties of polygalacturonase from Aspergillus niger MTCC 3323. Protein Expres Purif 87(1): 11-16.

3. Tapre AR, Jain RK (2014) Pectinases: Enzymes for fruit processing industry. Int Food Res J 21(2): 447-453.

4. Mojsov K, Ziberoski J, Bozinovic Z, Petreska M (2011) Comparison of effects of three commercial pectolytic enzyme preparations in white winemaking. Appl Technol Innov 4(1): 34-38.

5. Laaksonen O, Sandell M, Nordlund E, Heiniö R, Malinen H, et al. (2012) The effect of enzymatic treatment on blackcurrant (Ribes nigrum) juice flavour and its stability. Food Chem 130: 31-41.
6. Alimardani-Theuil P, Gainvors-Claisse A, Duchiron F (2011) Yeasts: an attractive source of pectinases- from gene expression to potential applications: a review. Process Biochem 46: 1525-1537.

7. Blandino A, Dravillas K, Cantero D, Pandiella SS, Webb C (2001) Utilization of whole wheat flour for the production of extracellular pectinases by some fungal strains. Process Biochem 37: 497-503.

8. Demir H, Tari C (2014) Valorization of wheat bran for the production of polygalacturonase in SSF of Aspergillus sojae. Ind Crop Prod 54: 302309.

9. Blanco P, Sieiro C, Villa TG (1999) Production of pectic enzymes in yeasts. FEMS Microbiol Lett 175: 1-9

10. Gainvors A, Frézier V, Lemaresquier H, Lequart C, Aigle M, et al. (1994) Detection of polygalacturonase, pectin-lyase and pectin-esterase activities in a Saccharomyces cerevisiae strain. Yeast 10:1311-1319.

11. Schwan RF, Wheals AE (2004) The microbiology of cocoa fermentation and its role in chocolate quality. Crit Rev Food Sci 44: 205-222.

12. Fernández-González M, Úbeda JF, Vasudevan TG, Cordero Otero RR, Briones AI (2004) Evaluation of polygalacturonase activity in Saccharomyces cerevisiae wine strains. FEMS Microbiol Lett 237(2): 261-266.

13. Jia JH, Wheals A (2000) Endopolygalacturonase genes and enzymes from Saccharomyces cerevisiae and Kluyveromyces marxianus. Curr Genet 38: 264-270.

14. Favela-Torres E, Volke-Sepulveda T, Viniegra-González G (2006) Production of hydrolytic depolymerizing pectinases. Food Technol Biotech 44: 221-227.

15. Tari C, Gogus N, Tokatli F (2007) Optimization of biomass, pellet size and polygalacturonase production by Aspergillus sojae ATCC 20235 using response surface methodology. Enzyme Microb Tech 40: 11081116. 
16. Kouhoundé SHS, Adéoti K, Delvigne F, Savadogo A, Traore AS, et al. (2014) The use of microorganisms of cassava retting for the production of pectinolytic enzymes. J Microb Biotech Food Sci 4: 277-281.

17. Gognies S, Simon G, Belarbi A (2001) Regulation of the expression of endopolygalacturonase gene PGU1 in Saccharomyces. Yeast 18(5): 423-432.

18. Radoi F, Kishida M, Kawasaki H (2005) Endo-polygalacturonase in Saccharomyces wine yeasts: effect of carbon source on enzyme production. FEMS Yeast Res 5: 663-665.

19. Winborne MP, Richard AD (1978) Pectinolytic activity of Saccharomyces fragilis in controlled environments. Biotechnol Bioeng 20: 231-242.

20. Nakagawa T, Miyaji T, Yurimoto H, Sakai Y, Kato N, et al. (2000) A Methylotrophic Pathway Participates in Pectin Utilization by Candida boidinii. Appl Environ Microbiol 66(10): 4253-4257.

21. Schwan RF, Rose AH (1994) Polygalacturonase production by Kluyveromyces marxianus: effect of medium composition. J Appl Bacteriol 76: 62-67.

22. Dawes IW, Hardie ID (1974) Selective killing of vegetative cells in sporulated yeast cultures by exposure to diethyl ether. Mol Gen Genet 131(4): 281-289.

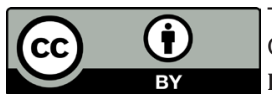

This work is licensed under Creative Commons Attribution 4.0 License

DOI: 10.19080 /AIBM.2017.03.555608
23. McKay AM (1988) A plate assay method for the detection of fungal polygalacturonase secretion. FEMS Microbiol Lett 56: 355-358.

24. Miller GL (1959) Use of dinitrosalicylic reagent for determination of reducing sugars. Anal Chem 31: 426-428.

25. Serrat M, Bermúdez RC, Villa TG (2002) Production, purification, and characterization of a polygalacturonase from a new strain of Kluyveromyces marxianus isolated from coffee wet- processing wastewater. Appl Biochem Biotech 97:193-208.

26. Masoud W, Jespersen L (2006) Pectin degrading enzymes in yeasts involved in fermentation of Coffea Arabica in East Africa. Int J Food Microbiol 110(3): 291-296.

27. Cruz-Guerrero A, Bárzana E, García-Garibay M, Gómez-Ruiza L (1999) Dissolved oxygen thresholdfor the repression of endopolygalacturonase production by Kluyveromyces marxianus. Process Biochem 34: 621-624.

28. Martens-Uzunova ES, Schaap PJ (2008) An evolutionary conserved D-galacturonic acid metabolic pathway operates across filamentous fungi capable of pectin degradation. Fungal Genet Biol 45: 1449-14457.

\section{Your next submission with Juniper Publishers} will reach you the below assets

- Quality Editorial service

- Swift Peer Review

- Reprints availability

- E-prints Service

- Manuscript Podcast for convenient understanding

- Global attainment for your research

- Manuscript accessibility in different formats

( Pdf, E-pub, Full Text, Audio)

- Unceasing customer service

Track the below URL for one-step submission https://juniperpublishers.com/online-submission.php 